\title{
Evaluación y caracterización de compuestos bioactivos en sacha manzano (Hesperomeles escalloniifolia Schltdl.) y upatankar (Berberis boliviana L).
}

\author{
Melquiades Barragán Condori ${ }^{*}$, Juan Marcos Aro $\mathrm{Aro}^{2}$, Victor Justiniano Huamaní Meléndez ${ }^{1}$, María del \\ Carmen Delgado Laime ${ }^{3}$, Claudia Palomino Sihuipauccar ${ }^{1}$, Luis David Barragán Jove ${ }^{4}$ \\ ${ }^{1}$ Departamento de Ciencias Básicas, Universidad Nacional Micaela Bastidas de Apurímac \\ 2 Departamento de Agroindustrias, Universidad Nacional del Altiplano - Puno. \\ ${ }^{3}$ Universidad Nacional José María Arguedas - Apurímac. \\ ${ }^{4}$ Universidad Nacional de San Antonio Abad del Cusco.
}

Recibido el 14 de noviembre del 2017, aceptado el 23 de diciembre del 2017

DOI: https://doi.org/10.33017/RevECIPeru2017.0012/

\section{Resumen}

Se evaluó y caracterizó compuestos bioactivos en dos frutos nativos denominados sacha manzano (Hesperomeles escalloniifolia S) y upatankar (Berberis boliviana L), para lo cual se extrajo los pigmentos antociánicos con metanol acidificado al $0.01 \%$, se filtró y concentró en rotavapor, luego se centrifugó, decantó y aforó a un volumen conocido para su posterior análisis, purificación y evaluación. En el extracto se evaluó el contenido de antocianinas totales (CAT) por el método de $\mathrm{pH}$ diferencial, polifenoles totales (PFT) por el método Folin-Ciocalteu. Seguidamente se purificó el extracto con cartucho set pak C-18 y en esta solución se identificó los espectros de antocianinas mediante FTIR y UV Visible. Los extractos caracterizados por espectrofotometría UV-Vis presentan valores máximos de absorbancia a $520 \mathrm{~nm}$ que corresponden a estructuras antociánicas ya establecidas, del mismo modo por espectroscopia FTIR-ATR se observan fuertes señales de absorción a diferentes valores de frecuencias de vibración $\left(\mathrm{cm}^{-1}\right)$ que corresponden a grupos funcionales de antocianinas. Los valores de CAT son de 978.83 y 75.39 (mg de cianidina 3-glucosido/100 g de peso fresco) del PFT 4902.41 y 1029.59 ( $\mathrm{mg}$ de ácido gálico/100 g de peso fresco) para upatankar y sacha manzano respectivamente. Los espectros UV Vis y FTIR corresponden a antocianinas. El alto contenido de CAT y PFT del upatankar nos permite recomendar su uso como antioxidante en alimentos funcionales y extractos naturales en la agroindustria.

Descriptores: Antocianinas, polifenoles, antioxidantes, radicales libres, flavonoides.

\begin{abstract}
Bioactive compounds were evaluated and characterized in two native fruits called sacha manzano (Hesperomeles escalloniifolia S) and upatankar (Berberis boliviana $\mathrm{L}$ ), for which the anthocyanin pigments were extracted with acidified to $0.01 \%$ methanol, filtered and concentrated in a rotary evaporator. centrifuged, decanted and adjusted to a known volume for further analysis, purification and evaluation. In the extract the total anthocyanin content (CAT) was evaluated by the differential pH method, total polyphenols (PFT) by the Folin Ciocalteu method. The extract was then purified with set pak cartridge C-18 and in this solution the anthocyanin spectra were identified by FTIR and Visible UV. The extracts characterized by UV-Vis spectrophotometry have maximum absorbance values at $520 \mathrm{~nm}$ corresponding to established anthocyanin structures, and FTIR-ATR spectroscopy shows strong absorption signals at different vibration frequency values $(\mathrm{cm}-1)$ which correspond to functional groups of anthocyanins. The CAT values are 978.83 and 75.39 (mg of cyanidin 3-glucoside / 100 $\mathrm{g}$ of fresh weight) of PFT 4902.41 and 1029.59 ( $\mathrm{mg}$ of gallic acid / $100 \mathrm{~g}$ of fresh weight) for upatankar and sacha manzano tree respectively. The UV Vis and FTIR spectra correspond to anthocyanins. The high content
\end{abstract}


of CAT and PFT of upatankar allows us to recommend its use as an antioxidant in functional foods and natural extracts in the agroindustry.

Keywords: Anthocyanins, polyphenols, antioxidants, free radicals, flavonoids.

\section{Introducción}

La biodiversidad en frutos nativos de la Región Apurímac por su enorme variabilidad de ecosistemas producto de su ubicación geográfica, presenta frutos nativos como el sacha manzano (Hesperomeles escalloniifolia $S$ ) y el upatankar (Berberis boliviana $L$ ), los mismos que requieren evaluar sus compuestos bioactivos. Diversas investigaciones, han confirmado los beneficios para la salud que aporta la ingesta de frutas y verduras ricas en antioxidantes, principalmente en la reducción de enfermedades cardiovasculares [1] (Sadani \& Nadkarni, 1996), efecto protector en la prevención de procesos degenerativos de enfermedades cancerígenas, mostrar actividad antioxidante, atrapar radicales y actuar como agentes quimioprotectores.

Se plantea la hipótesis de que los frutos nativos pigmentados de color rojo poseen compuestos fenólicos y antocianinas.

El objetivo general de éste trabajo de investigación es caracterizar y cuantificar compuestos fenólicos y antocianinas en frutos nativos sacha manzano y upatankar.

Los objetivos específicos son:

Caracterizar por espectroscopia UV-Vis, FTIR-ATR compuestos bioactivos en frutos nativos de la región Apurímac.

Determinar el contenido de antocianinas totales CAT en frutos nativos de la región Apurímac.

Determinar el contenido de polifenoles PFT totales en frutos nativos de la región Apurímac.

\section{Metodología}

Las frutos nativos de la región Apurímac fueron recolectados en la Provincia de Abancay distrito de Circa (Figuras 1y 2).

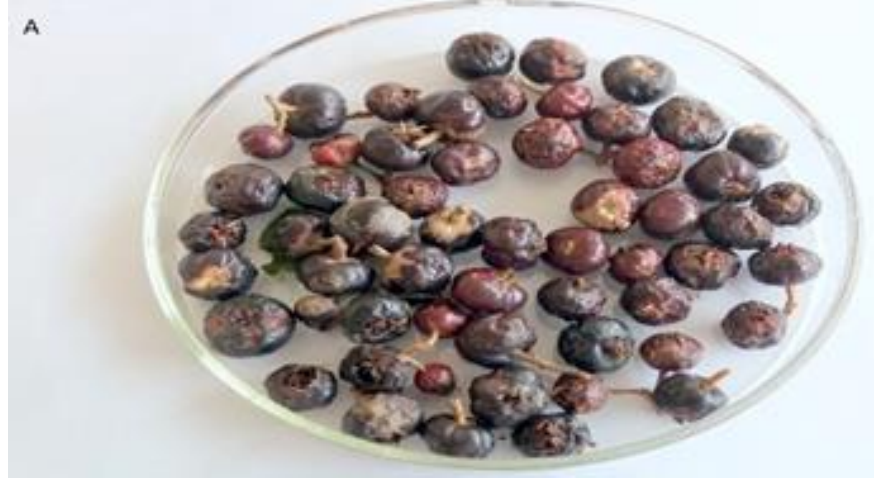

Figura 1: Fruto nativo sacha manzano (Hesperomeles escalloniifolia $S$.)

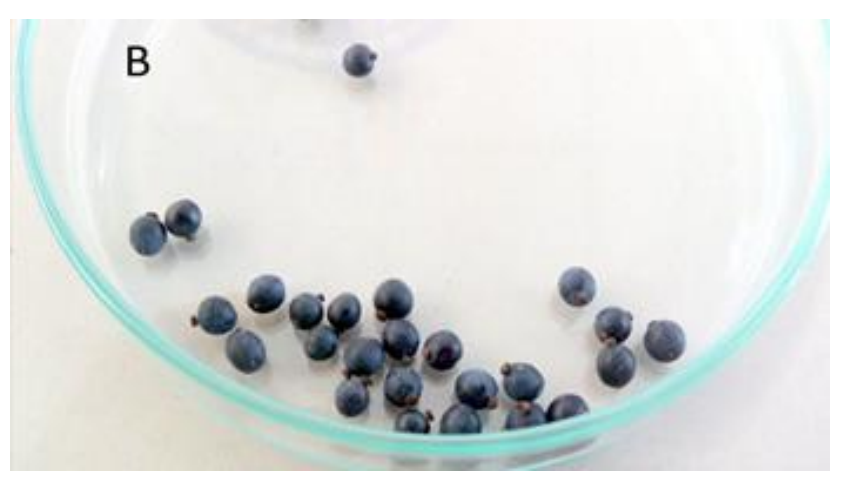

Figura 2: Fruto nativo upatankar (Berberis boliviana L)

\subsection{Extracción del pigmento}

Se extrajo los pigmentos antociánicos con metanol acidificado al $0.01 \%$, se filtró, concentró en rotavapor, luego se centrifugó, decantó y aforó a un volumen conocido para su posterior análisis, purificación y evaluación. (Del Carpio Jiménez, Serrano Flores, \& Giusti, 2009) [2].

\subsection{Espectrofotometría UV -Visible}

Los espectros se leyeron en un rango de longitud de onda de 190 a $1100 \mathrm{~nm}$ en un espectrofotómetro THERMO GENESYS 10S UV VIS en cubetas de cuarzo de $10 \mathrm{~mm}$ de espesor con velocidad de registro de $2500 \mathrm{~nm} / \mathrm{min}$ y se utilizó metanol como solvente (Castillo et al., 2010) [3].

\subsection{Espectrometría FTIR-ATR}


Los espectros se realizaron en un intervalo de 4000 a $600 \mathrm{~cm}-1$ y en unidades de transmitancia, en un Espectrómetro FTIR - ATR Nicolet ${ }^{\mathrm{TM}}$ iS-10. El procesamiento de los espectros se realizó mediante el Software OMNI. Las muestras se midieron utilizando el aditamento de ATR (Castillo et al., 2010) [3].

\subsection{Contenido de antocianinas totales (CAT)}

El contenido de antocianinas totales se determinó según el método de $\mathrm{pH}$ diferencial, descrito (GIUSTI \& WROLSTAD, 1996) [4] usando dos sistemas buffer: cloruro de potasio $(\mathrm{KCl})$; pH 1,0 ( 0,025 M ), y acetato de sodio $(\mathrm{CH} 3 \mathrm{COONa}), \mathrm{pH} 4,5(0,4 \mathrm{M})$.

\subsection{Análisis de polifenoles totales (PFT)}

La concentración de fenoles totales en extractos fue medida por espectrofotometría, basándose en una reacción colorimétrica de óxido-reducción. El agente oxidante utilizado fue el reactivo de FolinCiocalteu, descrito por (Singleton, Orthofer, \& Lamuela-Raventos, 1999) [5].

\subsection{Análisis estadístico}

El análisis de resultados se procesaron mediante un análisis de varianza y las medias fueron comparadas con la prueba de Tukey con una significancia de 0.05 usando el software estadístico INFOSTAT Versión 2013 (Di Rienzo et al., 2011) [6]. Los resultados se observan en las tablas de resultados de los diferentes métodos de análisis.

\section{Resultados}

De los espectros UV - Vis de las antocianinas

Los espectros UV - Visible de los extractos obtenidos pueden variar con el $\mathrm{pH}$ y solvente utilizados (Figuras 3 y 4 ).

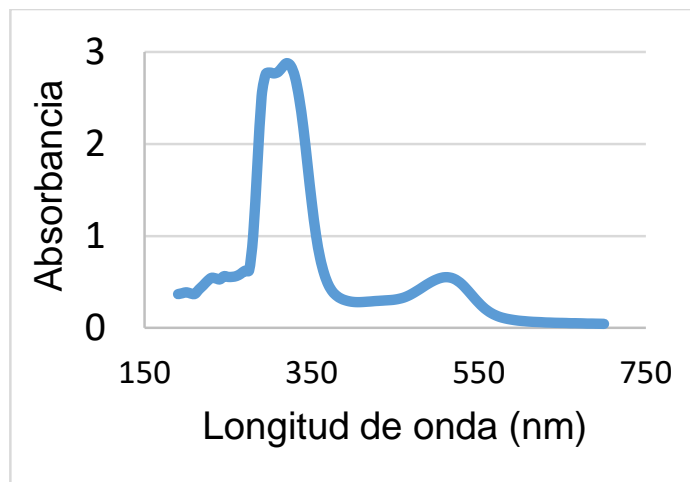

Figura 3: Espectros UV-Vis de antocianinas en frutos nativos de sacha manzano.

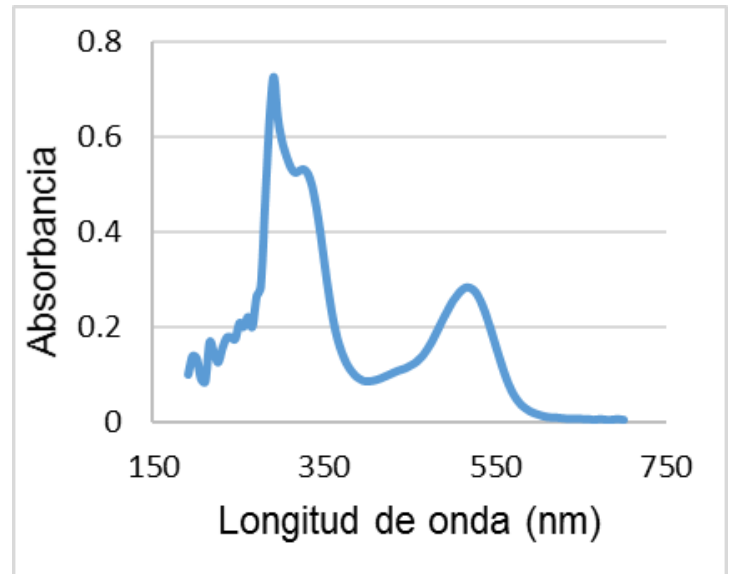

Figura 4: Espectros UV-Vis de antocianinas en frutos nativos de upatankar.

De los espectros FTIR - ATR de las antocianinas

Se determinó los espectros FTIR en extracto seco de frutos nativos en el espectrómetro infrarrojo con accesorio ATR, siendo los espectros resultantes las figuras 5 y 6 .

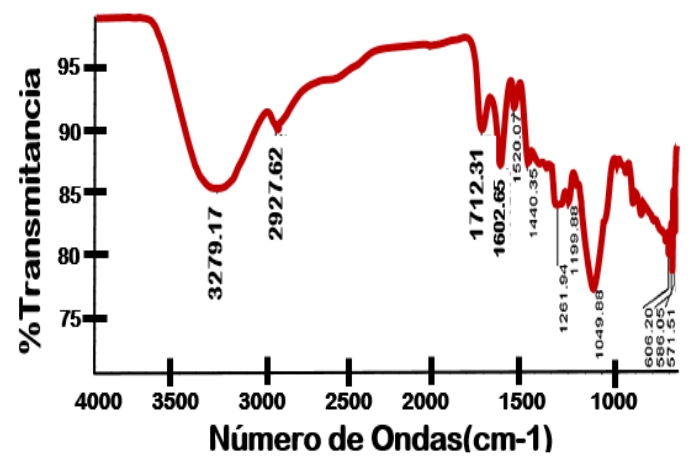

Figura 5: Espectros FTIR - ATR de antocianinas en frutos nativos de sacha manzano

De la cuantificación de antocianinas totales (CAT) por el método de $\mathrm{pH}$ diferencial

Los resultados de los análisis de contenido de antocianinas totales CAT se tienen en las tablas 1,2 y figura 7 . 
Revista ECIPerú

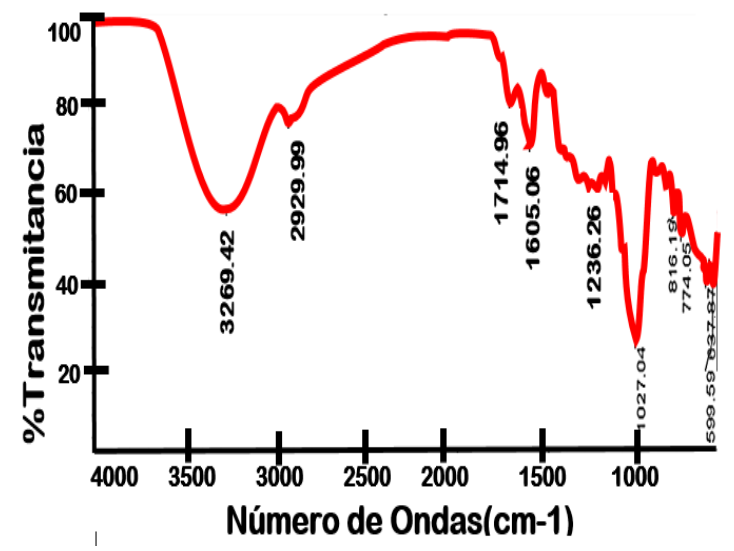

Figura 6: Espectros FTIR-ATR de antocianinas en frutos nativos de upatankar.

Tabla 1: Contenido de antocianinas totales CAT (mg cianidina 3-glucosido/100 de muestra fresca) en frutos nativos.

Tabla 1: Contenido de antocianinas totales CAT (mg cianidina 3-glucosido/100 de muestra fresca) en

\begin{tabular}{ll} 
Muestra & $\begin{array}{l}\text { PFT })(\mathrm{mg} \text { ácido frutos } \\
\text { gálico/100g) }\end{array}$ \\
\hline $\begin{array}{l}\text { Sacha manzana } \\
\text { Upatankar }\end{array}$ & $1029.59 \pm 17.61^{\mathrm{a}}$ \\
& $4902.41 \pm 60.63^{\mathrm{b}}$
\end{tabular}

nativos.

\begin{tabular}{ll}
\hline Muestra & $\begin{array}{l}\text { CAT }(\mathrm{mg} \text { cianidina 3 } \\
-\mathrm{G} / 100 \mathrm{~g})\end{array}$ \\
\hline Sacha manzano & $75.39 \pm 0.41^{\mathrm{a}}$ \\
Upatankar & $978.83 \pm 1.42^{\mathrm{b}}$
\end{tabular}

Datos

expresados en media $\pm S D, n=3, p<0.05$

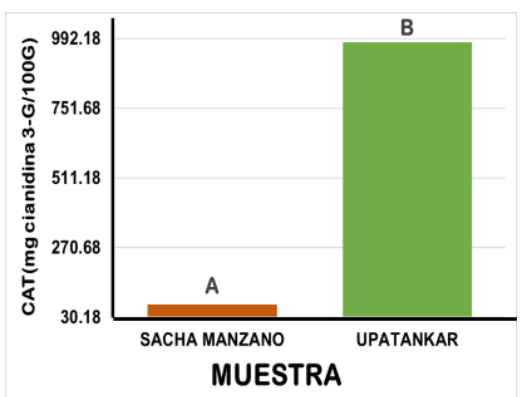

Figura 7: Contenido de antocianinas totales CAT (mg cianidina 3-glucosido/100 de muestra fresca) en frutos nativos.
Diciembre, 2017

Tabla 2: Diferencia significativa de Tukey para valores de CAT (mg cianidina 3-glucosido/100g)

\begin{tabular}{llll}
\hline Muestra & Medias & $\mathbf{n}$ & E.E. \\
\hline $\begin{array}{l}\text { Sacha } \\
\text { manzano }\end{array}$ & 75.39 & 3 & $0.61 \mathrm{a}$ \\
$\begin{array}{l}\text { Upatankar } \\
\text { Uptank }\end{array}$ & 978.83 & 3 & $0.61 \mathrm{~b}$ \\
\hline
\end{tabular}

Medias con una letra común no son significativamente diferentes $(p \geq 0.05)$.

Puesto que el valor - p es menor que 0.05, existe una diferencia estadísticamente significativa entre las medias de CAT (mg cianidina 3-glucosido/100) entre un nivel de muestra y otro, con un nivel del $95.0 \%$ de confianza.

De la cuantificación de Polifenoles totales (PFT) por el método Folin-Ciocalteu.

Los resultados de los análisis de contenido de polifenoles totales PFT se tienen en las tablas 3,4 y figura 8.

Tabla 3: Polifenoles totales (PFT) (mg ácido gálico/100g muestra fresca) en frutos nativos.

\begin{tabular}{ll}
\hline Muestra & $\begin{array}{l}\text { CAT } \\
\text { cianidina } \\
\text { g/100g) }\end{array}$ \\
\hline Sacha & $\begin{array}{l}\text { (mg } \\
\text { manzano }\end{array}$ \\
$\begin{array}{l}\text { Upatankar } \\
978.39 \pm 0.41^{\mathrm{a}}\end{array}$ \\
\hline xpresados en media $\pm \mathrm{SD}, \mathrm{n}=3, \mathrm{p}<0.05$
\end{tabular}

Datos

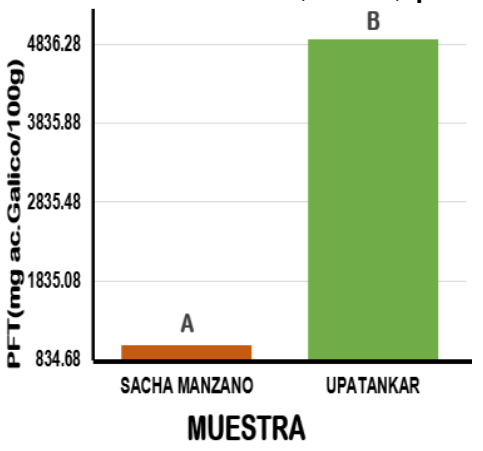

Figura 8: Polifenoles totales (PFT) (mg ácido gálico/100 muestra fresca) en frutos nativos.

Tabla 4: Diferencia significativa de Tukey para valores de PFT (mg ácido gálico/100g)

\begin{tabular}{llll}
\hline Muestra & Medias & n & E.E. \\
\hline $\begin{array}{l}\text { Sacha } \\
\text { manzano }\end{array}$ & 1029.59 & 3 & $25.77 a$ \\
& & &
\end{tabular}




$\begin{array}{llll}\text { Upatankar } \quad 4902.41 \quad 3 & 25.77 b\end{array}$

Medias con una letra común no son significativamente diferentes $(p \geq 0.05)$.

Puesto que el valor - p es menor que 0.05, existe una diferencia estadísticamente significativa entre las medias de PFT (mg ácido gálico/100g) entre un nivel de muestra y otro, con un nivel del $95.0 \%$ de confianza.

\section{Discusión de resultados}

\section{De los espectros UV - Vis de las antocianinas}

La posición de estas bandas según (Bedascarrasbure, Maldonado, Fierro Morales, \& Álvarez, 2006) [7] permite distinguir entre los distintos tipos de flavonoides, entre ellas las antocianinas que producen una banda entre 500$550 \mathrm{~nm}$ (Banda I), además de otras de menor intensidad entre $430-440 \mathrm{~nm}$ y $270-280 \mathrm{~nm}$ (Banda II), (Figura 9),
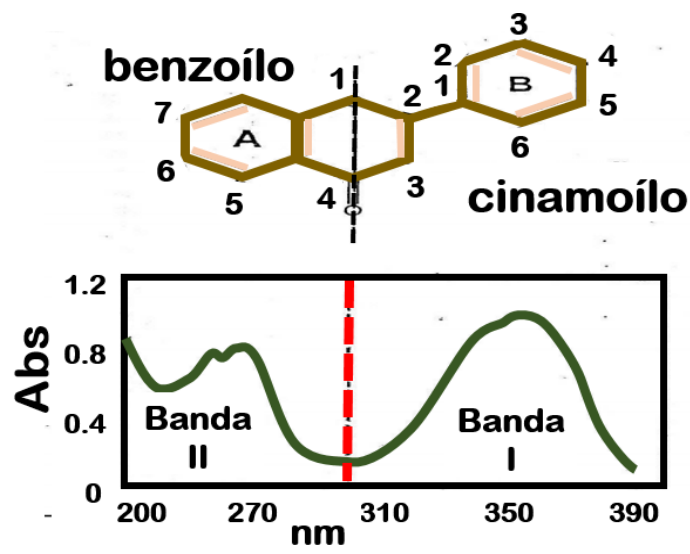

Figura 9: Bandas de absorción UV de flavonoides(Bedascarrasbure et al., 2006) [7].

Los espectros UV-Vis de los frutos nativos, presentan dos bandas de absorción máxima bien definidas. La banda I absorbe a una longitud promedio de $520 \mathrm{~nm}$, mientras que la banda II absorbe a una longitud promedio de $360 \mathrm{~nm}$ (Ver figuras 3 y 4 ).

Con estos resultados, ya se pueden hacer conjeturas preliminares de que los extractos podrían ser algún tipo de flavonoide en este caso del grupo de las antocianinas.

De los espectros FTIR - ATR de las antocianinas

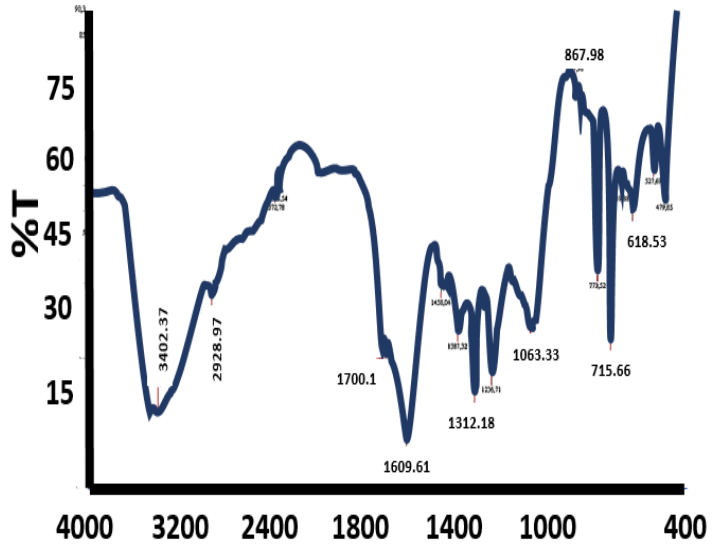

Figura 10: Espectro infrarrojo de la fracción de un colorante flavínico (Noriega Rivera, Coba Santamaría, Naikiai, \& Abad, 2011) [8].

En el espectro Infrarrojo FTIR-ATR (Figuras 5 y 6), la presencia de antocianinas puede ser interpretada de según diferentes autores como (Pavia, Lampman, Kriz, \& Vyvyan, 2008) [9, 10],(Torres et al., 2015).

Tensión O-H: Banda ancha desde 3500 a 3200 cm1. En ausencia de puentes de hidrógeno aparece como un pico agudo a $3650-3600 \mathrm{~cm}-1$.

El estiramiento $\mathrm{C}-\mathrm{H}$ se produce aproximadamente de 2900 a $3000 \mathrm{~cm}-1$. A medida que el átomo unido al carbono aumenta en masa, la frecuencia de vibración disminuye (los números de onda se hacen más pequeños).

Estiramiento $\mathrm{C}=\mathrm{C}$ anillo aromático a $1600-1475$ $\mathrm{cm}-1$.

1050 cm-1 se atribuye a la deformación en el plano aromático $=\mathrm{C}-\mathrm{H}$ (benceno).

Todas estas características de frecuencias de vibración sugieren una estructura similar a un flavonoide del tipo antocianina en los espectros FTIR-ATR de los frutos nativos sacha manzano y upatankar, todos estos espectros son semejantes al espectro flavínico Infrarrojo de la figura 10.

\section{De la cuantificación de antocianinas totales (CAT) por el método de $\mathrm{pH}$ diferencial}

En cuanto al contenido de antocianinas totales (CAT) se determinó 978.83 y 75.39 (mg de cianidina 3-glucosido/100 g de peso fresco) para upatankar y sacha manzano respectivamente. Investigaron el contenido de antocianinas para 5 especies del género Rubus determinado por el mismo método (Wada \& Ou, 2002) [11] y 4 de estas especies 
presentaron valores que van de 5.69 a $11.92 \mathrm{mg} / \mathrm{g}$ de materia seca y solo $R$. occidentalis presentó un mayor contenido de antocianinas $(34.65 \mathrm{mg} / \mathrm{g})$ que el encontrado en este estudio para $R$. adenotrichus (12.3 mg/g).

\section{De la cuantificación de Polifenoles totales (PFT) por el método Folin-Ciocalteu.}

En cuanto al contenido de polifenoles totales (PFT) resultó 4902.41 y 1029.59 (mg de ácido gálico/100 $\mathrm{g}$ de peso fresco) para upatankar y sacha manzano respectivamente. En investigaciones de papas nativas pigmentadas encontraron polifenoles totales (PFT) usando el método de Folin-Ciocalteu de 179.51 (mg ácido gálico/100g de muestra fresca) en papas de pulpa roja (Barragan Condori \& Aro Aro, 2017) [12], también (Kähkönen et al., 1999) [13], en muestras de remolacha (Beta vulgaris esculenta, 4,3 mg AGE/peso seco), tomate (Lycopersicum esculentum, 2,0 mg AGE/peso seco) y en el trebol rojo (red clover, 7,8 mg AGE/peso seco). De acuerdo a éstos resultados el fruto del upatankar, presenta mayor contenido de polifenoles totales siendo por ello un fruto muy prometedor por su capacidad antioxidante.

\section{Conclusiones}

Los espectros UV - Visible y FTIR corresponden a los espectros de antocianinas.

El fruto nativo upatankar presenta el más alto contenido en antocianinas totales frente al sacha manzano siendo este prometedor para uso como alimento funcional y para extractos como colorantes naturales.

En cuanto a polifenoles totales del mismo modo el upatankar presenta la mayor cantidad frente al sacha manzano.

\section{Agradecimientos}

Agradecer a la UNAMBA, por conceder al autor de este trabajo su año sabático por investigación y facilitar los materiales y equipos de laboratorio de la Escuela Académico Profesional de Ing. Agroindustrial para la ejecución de este trabajo de investigación.

\section{Referencias}

[1] G. R. Sadani, y G. D. Nadkarni, Role of tissue antioxidant defence in thyroid cancers. Cancer letters 109 (1996) 231235.

[2] C. del Carpio Jiménez, C. Serrano Flores, y M. Giusti, Caracterización de las antocianinas de los frutos de Berberis boliviana Lechler. Revista de la Sociedad Química del Perú 75 (2009) 76-86.

[3] G. Castillo, G. Michelena, C. Nogueiras, G. Ortega, D. Bello, M. Guerra, y G. Mieres, Caracterización cromatográfica y espectroscópica de un pigmento rojo obtenido a partir de Bothryodiplodia theobromae. ICIDCA Sobre los Derivados de la Caña de Azúcar 44 (2010) 15-20. Disponible en

http://www.redalyc.org/articulo.oa?id=223 120684003

[4] M. M. Giusti, R. E. Wrolstad, Characterization of red radish anthocyanins. Journal of Food Science 61 (1996) 322-326.

[5] V. L. Singleton, R. Orthofer, R. M. Lamuela-Raventos, Analysis of total phenols and other oxidation substrates and antioxidants by means of folinciocalteu reagent. Methods in enzymology 299 (1999) 152-178.

[6] J. A. Di Rienzo, F. Casanoves, M. G. Balzarini, L. Gonzalez, M. Tablada, y C. W. Robledo, InfoStat, Grupo InfoStat, FCA, Universidad Nacional de Córdova, Argentina, 2011.

[7] E. Bedascarrasbure, L. Maldonado, W. Fierro Morales, y A. Álvarez, Propóleos: Caracterización y normalización de propóleos argentinos. Revisión y actualización de composición y propiedades (Magna, Argetina, 2006)

[8] P. Noriega Rivera, P. Coba Santamaría, J. Naikiai, y J. Abad, Extracción, pruebas de estabilidad y análisis químico preliminar de la fracción colorante obtenida a partir del exocarpo del fruto de Renealmia Alpinia. LA GRANJA Revista de Ciencias de la Vida de la Universidad Politécnica Salesiana 13 (2011) 13-20. Disponible en: http://revistas.ups.edu.ec/index.php/ granja/article/view/13.2011.02/318

[9] D. L. Pavia, G. M. Lampman, G. S. Kriz, y J. A. Vyvyan, Introduction to spectroscopy (CENCAGE, 4ta Edición, 2008).

[10] R. Torres, G. Montero, B. Jaramillo, M. Beleño, L. Pérez, E. Romero, M. Coronado, Determinación del contenido energético de lignina obtenida a partir de vara de algodón del Valle de Mexicali, México. Memorias del XXXVI Encuentro 
Nacional de la AMIDIQ (2015) 2089-2094. Disponible en :

https://www.researchgate.net/publication/ 282329380 Determinacion del contenid o energetico de lignina obtenida a par tir de vara de algodon del Valle de $M$ exicai Mexico

[11] L. Wada, y B. Ou, Antioxidant activity and phenolic content of Oregon caneberries. Journal of Agricultural and Food Chemistry 50 (2002) 3495-3500.
[12] M. Barragan Condori, J. M. Aro Aro, Determinacion del efecto de procesos de coccion en papas nativas pigmentadas (Solanum tuberosum spp. andigena) sobre sus compuestos bioactivos. Revista de Investigaciones Altoandinas 19 (2007) 47-52.

[13] M. P. Kähkönen, A. I. Hopia, H. J. Vuorela, J. P. Rauha, K. Pihlaja, T. S. Kujala, y M. Heinonen, Antioxidant activity of plant extracts containing phenolic compounds. Journal of Agricultural and Food Chemistry 47 (1999) 3954-3962.

E-mail: melquim100@hotmail.com 